\title{
Current Solid Mechanics Research
}

\author{
Viggo Tvergaard
}

About thirty years ago James Lighthill wrote an essay on "What is Mechanics?" With that he also included some examples of the applications of mechanics. While his emphasis was on fluid mechanics, his own research area, he also included examples from research activities in solid mechanics.

Solid mechanics is a diverse science with roots in traditional research fields while also being an integral part of many entirely new areas. Much work is going on in classical research areas such as structural mechanics, where also many Ph.D.'s in solid mechanics use their expertise to solve complicated mechanics problems in private companies. This includes off-shore structures, large ships, modern bridges, and very tall buildings. Related to this is the analysis of large shell structures, and there is also a big demand for expertise on contact and friction mechanics, which are important research areas with many unresolved issues. Solid mechanics borders to electronics, medical devices, the energy sector, transportation, materials science, physics, and biology.

In the following a few examples are given of larger hot research areas that currently play an important role in solid mechanics research.

Materials mechanics. The classical nonlinear material models describe elastic-plastic deformations of solids or creep at high temperatures. These models are extended in many different directions. One is to account for damage evolution in the material and the effect of this damage on the stress-strain relations for the material. This can be small voids or micro-cracks or the evolution of failure between different parts of a multi-component material, such as a composite. In many of these application areas large deformations occur, so that the models must be developed in the context of general continuum mechanics. Size effects in

\footnotetext{
V. Tvergaard $(* 1943)$

Danish Technical University, Lyngby, Denmark

(C) The Author(s) 2016

P. Eberhard and S. Juhasz (eds.), IUTAM,

DOI 10.1007/978-3-319-31063-3_2
} 
material response is a very active sub-area here, that has spawned numerous research articles. It turns out that materials often have a characteristic length scale, such that the behavior depends on the size of the solid studied. A number of different mathematical formulations of material models are currently proposed that incorporate effects of the size in different ways.

Fracture mechanics is a subject of central importance in the description of material behavior. This involves singular crack-tip fields, determination of threshold values of crack parameters below which cracks will not grow, and studies of crack growth both in brittle solids and ductile solids or in solids where different kinds of damage develops and interacts with a macroscopic crack. Research in this area often makes use of cohesive zone models for the formulation of fracture criteria. Many solids are subjected to cyclic loading where fatigue fracture is the predominant failure mechanism that attracts much research interest.

Research on the mechanics of materials requires detailed knowledge of basic material behavior and therefore some of this research occurs in close collaboration with materials scientists. Recent interest in smaller and smaller length scales has opened research in nano-mechanics and molecular dynamics, where the research interfaces with materials physics.

Computational mechanics. As in many sciences, progress in solid mechanics research is closely tied to the fast development of computers. Problems in continuum mechanics often involve the solution of systems of nonlinear partial differential equations, which is possible only due to computers. Finite difference approximations can be used, but in solid mechanics variational formulations leading to finite element approximations are usually found most efficient. Computational mechanics is not just a separate research field, it is an integral part of most of the research in solid mechanics. To do this on a high level, as is needed, the researcher must have a deep insight in mathematical methods of numerical analysis, in algorithms, and in current developments in computer capabilities. And still the purpose of the solid mechanics researcher is not the development of these advanced methods in themselves, but the use of these methods to obtain new insight in solutions of complex problems in solid mechanics.

Dynamics. This important research area has its origin in vibration theory, used to avoid resonance failure in structures. Current research involves multibody and vehicle dynamics, chaotic behavior of dynamical systems, the dynamics and control of morphing structures, and friction induced vibrations. There are also relations to mechatronics such as the use of active or semi-active dampers to control instabilities in rotor dynamics. Methods of particle dynamics are developed to represent certain effects in dynamical systems.

Dynamics also includes wave propagation, which plays an important role in a number of applications, such as impact in vehicle collisions, dynamic crack growth, or fault propagation during earth quakes.

Instabilities in structures. This area started already with Euler's analyses of column instabilities. The understanding was much improved in the middle of the 20th century by the development of asymptotic methods for post-bifurcation behavior and associated methods for the evaluation of imperfection sensitivity. 
Subsequently, mathematical theories for bifurcation and post-bifurcation in elastic-plastic structures were developed. This has led to a lively area of research in structural instabilities, including subjects such as buckling localization, instabilities in thin surface layers where compressive stresses develop, and instabilities in metal foam.

Biomechanics. The use of mechanics to understand biological systems is a research area in rapid evolution. Within solid mechanics one area is bone mechanics. Here researchers need to incorporate typical features of living material where properties depend on and develop due to the applied stress fields.

For soft biological materials the constitutive models applied are strongly related to models developed for other soft materials, such as rubber, polymers or gels. This allows for numerical studies of a disease like aortic aneurysms, where localized arterial expansion can be life-threatening, or red blood cells subject to malaria, where the mechanics of cell deformation is central. The soft biological materials typically undergo large deformations, requiring general continuum mechanics in the formulations.

Also, the mechanical properties of cells attract much interest. Here cross-linked networks of the protein actin are important building blocks for the cytoskeleton. The behavior is somewhat related to polymers, and some researchers build this into continuum models that can be treated by the methods of mechanics. To this area belong also new developments in drug delivery systems.

Manufacturing. Metal forming analyses have developed strongly in the last couple of decades from the classical use of simple, highly approximate, rigid-plastic methods to the use of modern computational tools in solid mechanics. It is characteristic in these applications, like rolling, metal cutting or forging, that very large strains develop which must be accounted for in the mechanics formulations to be used for the numerical solutions. There are also cases where the size of the specimens formed is so small that size effects have to be accounted for in the constitutive models.

Additive manufacturing, or 3D printing, is a new process that offers interesting possibilities of near net shape forming in very complex geometries. There is focus on better understanding the material properties obtained in this type of process, and the important effect of unavoidable residual stresses.

Optimal design. Optimization of the properties is an important goal for all engineering structures. This can mean minimum weight for a given functionality, maximum strength for a given amount of material, or minimum stress concentration to avoid fatigue. Systematic research in this area has been boosted enormously by the development of optimization algorithms such as linear programming or quadratic programming, which allow for efficient computer solutions with many degrees of freedom. Much of the current research is focused on topology optimization, which uses homogenization theory together with optimization algorithms to find optimal configurations with few initial restrictions. These methods are also used to optimize compliant mechanisms, MicroElectroMechanical Systems (MEMS), and materials with extremal properties. 
Open Access This chapter is distributed under the terms of the Creative Commons AttributionNoncommercial 2.5 License (http://creativecommons.org/licenses/by-nc/2.5/) which permits any noncommercial use, distribution, and reproduction in any medium, provided the original author(s) and source are credited.

The images or other third party material in this chapter are included in the work's Creative Commons license, unless indicated otherwise in the credit line; if such material is not included in the work's Creative Commons license and the respective action is not permitted by statutory regulation, users will need to obtain permission from the license holder to duplicate, adapt or reproduce the material. 